\title{
Burst Synchronization in Two Pulse-Coupled Resonate-and-Fire Neuron Circuits
}

\author{
Kazuki Nakada ${ }^{1}$, Tetsuya Asai ${ }^{2}$ and Hatsuo Hayashi ${ }^{1}$ \\ 1 Kyushu Institute of Technology nakada@brain.kyutech.ac.jp \\ ${ }^{2}$ Hokkaido University asai@sapinens-ei.eng.hokudai.ac.jp
}

The present paper addresses burst synchronization in out of phase observed in two pulse-coupled resonate-and-fire neuron (RFN) circuits. The RFN circuit is a silicon spiking neuron that has second-order membrane dynamics and exhibits fast subthreshold oscillation of membrane potential. Due to such dynamics, the behavior of the RFN circuit is sensitive to the timing of stimuli. We investigated the effects of the sensitivity and the mutual interaction on the dynamic behavior of two pulse-coupled RFN circuits, and will demonstrate out of phase burst synchronization and bifurcation phenomena through circuit simulations.

\section{Introduction}

Functional networks of silicon spiking neurons are shown to be useful in a wide variety of applications [5]-[10]. Recent research efforts have concentrated on realtime event-based computation, so-called temporal cording, in which coincidence or synchrony detection plays essential roles in neural information processing, such as auditory perception [6], onset detection [8], and learning and memory [9]-[10]. Temporal filtering properties are also significant to extract temporal structure of spike sequences in which information may be encoded.

Computational performance of such functional networks of silicon spiking neurons will be limited if their components are quite simple. For instance, the Axon-Hillock circuit [3], widely known as an electronic analogue of the integrateand-fire neuron (IFN) model, can only act as a low-pass filter for a sequence of pulses. For increasing their performance, alternative silicon spiking neurons [14]-[21], such as a low-power IFN circuit with frequency adaptation [17] and asynchronous chaotic spiking neuron circuits [19], [20], have been previously developed. These circuits increase selectivity to inputs into a silicon spiking neuron due to synchrony detection and temporal filtering properties. Synaptic circuits with short-term plasticity [8], [10]-[12] and spike-timing-dependent plasticity [10], [13] can also increase computational performance of silicon spiking neural networks. However, they can work effectively only at network level.

In this context, we designed an analog integrated circuit for a resonate-andfire neuron (RFN) model [1] using complementary metal-oxide-semiconductor (CMOS) technology [21]. The RFN model is a spiking neuron model that has second-order membrane dynamics and exhibits damped subthreshold oscillation

Please use the following format when citing this chapter:

Nakada, K., Asai, T., Hayashi, H., 2006, in IFIP International Federation for Information Processing, Volume 218, Professional Practice in Artificial Intelligence, eds. J. Debenham, (Boston: Springer), pp. 285-294. 
of the membrane potential. Due to such membrane dynamics, the RFN model is sensitive to the timing of stimuli, and thus it exhibits nonlinear phenomena observed in biological neurons, such as coincidence detection, post-inhibitory rebound, and frequency preference [1]. The RFN circuit acts as a coincidence detector and a band-pass filter at circuit level since it inherits the sensitivity of the RFN model. Thus, it is expected to be useful for large-scale implementation of functional silicon spiking neural networks.

In the present work, we focus on dynamic behavior of a system of two pulsecoupled RFN circuits. It is expected that pulse-coupled RFN circuits exhibit different behavior as compared to pulse-coupled IFN circuits because of the peculiar sensitivity to the timing of inputs of the RFN circuit. As a first step toward understanding of collective behavior of the coupled RFN circuits, we investigated how effective the sensitivity and the mutual interaction were to the behavior of the two pulse-coupled RFN circuits. Through circuit simulations using the simulation program with integrated circuit emphasis (SPICE), we will demonstrate out of phase synchronization of periodic and quasi periodic bursts, and bifurcation phenomena.

\section{Burst Synchronization in Two Pulse-Coupled Resonate-and-Fire Neuron Models}

Let us here summarize a resonate-and-fire neuron (RFN) model [1], and burst synchronization in out of phase observed in two pulse-coupled RFN models.

\subsection{Resonate-and-fire neuron model}

The RNF model is a spiking neuron model that has second-order subthreshold membrane dynamics and a firing threshold [1]. The dynamics of the RFN model are described by:

$$
\begin{aligned}
& \dot{x}=b x-w y+I \\
& \dot{y}=w x+b y
\end{aligned}
$$

or by an equivalent complex form:

$$
\dot{z}=(b+i w) z+I
$$

where $z=x+i y$ is a complex variable. The real and imaginary parts, $x$ and $y$, are the current- and voltage-like variables, respectively. The constants $b$ and $w$ are parameters, and $I$ is an external input. If the variable $y$ exceeds a certain threshold $a_{\mathrm{th}}$, the variable $z$ is reset to an arbitrary value $z_{o}$, which describes activity-dependent after-spike reset. This model has second-order membrane dynamics, and thus it exhibits dynamic behavior, such as fast subthreshold oscillation, resulting in the coincident detection, post-inhibitory-rebound, and frequency preference [1]. 

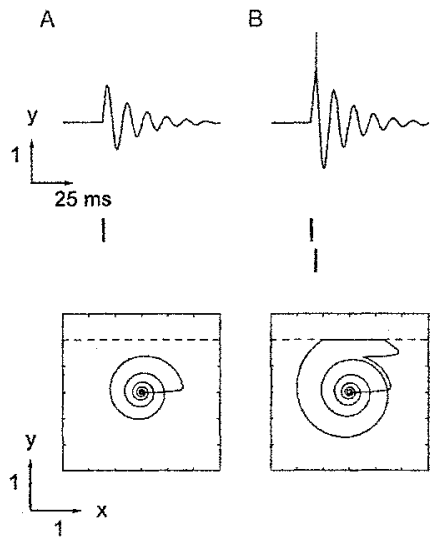

C

1

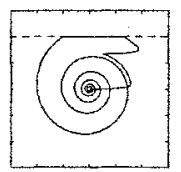

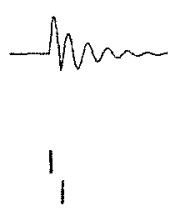

D
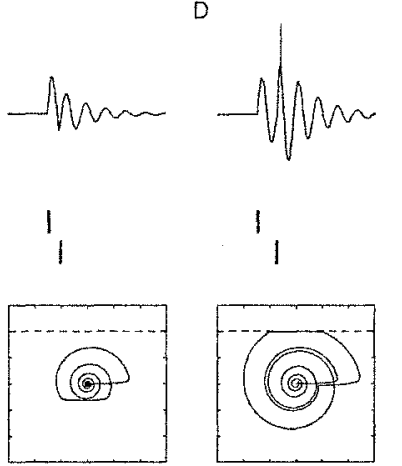

$E$

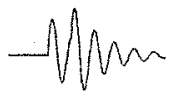

1

input pulses

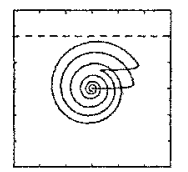

Fig. 1. Behavior of the resonate-and-fire neuron model in response to (A) a single pulse, (B) a coincident doublet, (C) a non-resonant doublet, (D) a resonant doublet, and $(E)$ a non-resonant doublet, where output pulses are manually written.
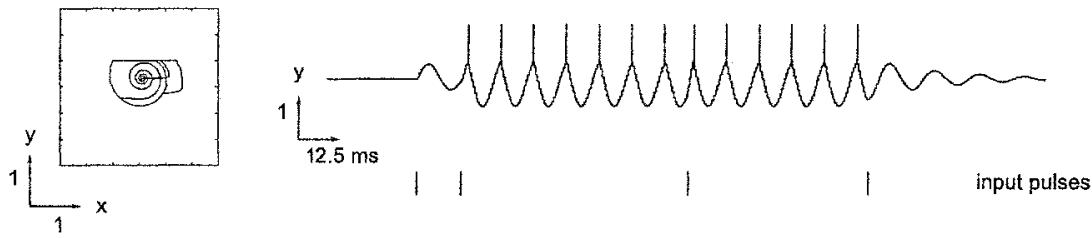

Fig. 2. Pacemaker activity of the resonate-and-fire neuron model as a bias current is injected. Output pulses are manually written. The last pulse cancels out the pacemaker activity.

Figure 1 shows typical behavior of the RFN model in response to input pulses. In this case, we used the following parameters: $b=-0.1, w=1, a_{t h}=i$, $z_{0}=-0.5+i$, and the input pulses were given by: $I=i_{\max }(t / \tau) \exp (1-t / \tau)$, where the time constant $\tau=0.05 \mathrm{~ms}$, the maximal amplitude $i_{\max }=12$, and $t$ was an elapsed time since a pulse was given. When a pulse that cannot evoke an action potential alone arrives at an RFN model, a damped subthreshold oscillation occurs, as shown in Fig. 1A. When a pair of pulses arrives at the RFN model at a short interval (a coincident doublet, e.g. $2.5 \mathrm{~ms}$, Fig. 1B), or at an interval nearly equal to the intrinsic period of the subthreshold oscillation (a resonant doublet, $12.5 \mathrm{~ms}$, Fig. 1D), the RFN model fires a spike. However, the RFN model does not fire a spike in response to a doublet with an interval in other ranges, e.g. $7.5 \mathrm{~ms}$ (Fig. 1C) or $15.0 \mathrm{~ms}$ (Fig 1E).

The RFN model fires a sequence of spikes in response to inputs when a bias current is injected (pacemaker activity), as shown in Fig. 2, where the bias current $i_{b i i s}=0.68$ and the maximal amplitude of the input pulses $i_{\max }=5$. Depending on the timing of input pulses, the pacemaker activity disappears 
A

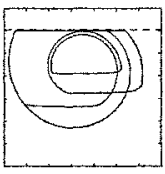

B

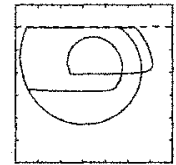

c

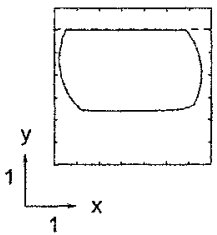

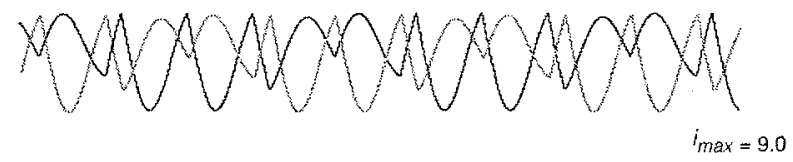
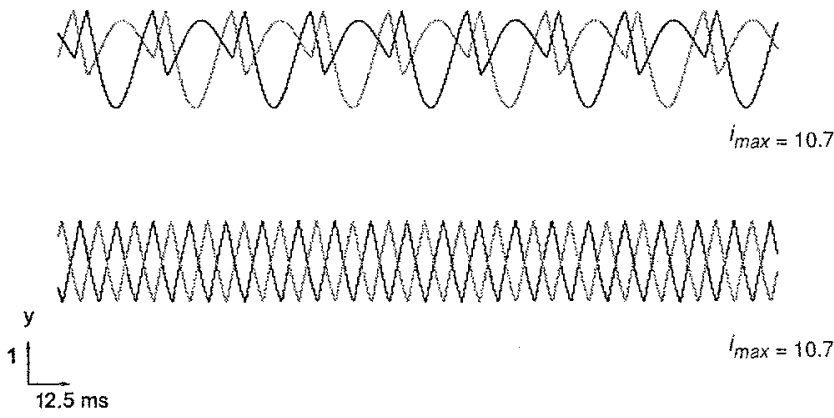

Fig. 3. Out of phase synchronization of bursts in two pulse-coupled resonate-and-fire neuron models. (A) $3: 3$ phase-locking $\left(i_{\text {max }}=9.0\right.$ ), (B) $2: 2$ phase locking $\left(i_{\text {max }}=10.7\right)$, and $(C)$ 1:1 phase locking $\left(i_{\max }=10.7\right)$, where light and dark waveforms were obtained from the neurons, 1 and 2 , respectively.

(Fig. 2 right). This implies that even two coupled RFN models exhibit complex phenomena due to the sensitivity to the input timing and mutual interaction.

\subsection{Burst synchronization in two pulse-coupled RFN models}

We here consider a system of two pulse-coupled RFN models, which is given by the following equation:

$$
\dot{z}_{j}=(b+i w) z_{j}+i_{\text {bias }}+i_{\max }\left(t_{k} / \tau\right) \exp \left(1-t_{k} / \tau\right)(j, k=1,2)
$$

where $z_{j}=x_{j}+i y_{j}$ is a complex state variable of the $j$-th neuron. The real and imaginary parts, $x_{j}$ and $y_{j}$, are the current- and voltage-like variables, respectively. The constants $b$ and $w$ are parameters, and $i_{b i a s}$ is a bias current. The time constant $\tau=0.05 \mathrm{~ms}$ and the maximal amplitude $i_{m a x}$, and $t_{k}$ is an elapsed time since the arrival of the latest spike from another neuron $k$. In the following, the threshold $a_{t h}$, and the constants $b$ and $w$ have the same values as those used in the previous section, respectively.

The system of two pulse-coupled RFN models has been previously analyzed in view of anti-phase synchronization and its stability [2]. However, the effects of the reset value $z_{o}$ on such synchronization were not considered in the previous work. The reset value may affect the sensitivity to the timing of input to the RFN model. Although any positive input given on the right side of the phase 
A

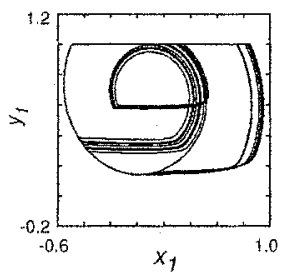

B

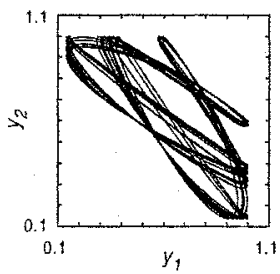

Fig. 4. Out of phase synchronization of quasi periodic bursts in two pulse-coupled resonate-and-frie neuron models.

plane act as excitatory, it is determined whether a positive input given on the left side of the phase plane act as excitatory or not depending on the strength and the phase of the input, as shown in Fig. 1. The reset value determines the trajectory of the RFN model after firing, resulting in changing the sensitivity to the timing of input.

For increasing the effects on the sensitivity to the timing of inputs to the RFN model, we set the reset value at $z_{0}=-0.5+i$. Furthermore, we set the bias input at $i_{\text {brias }}=0.68$ so that each RFN model became a pacemaker. Under such conditions, we investigated the behavior of two pulse-coupled RFN models and the effects of the sensitivity and the mutual interaction between the two RFN models. We changed the maximal amplitude of the pulse input $i_{\text {max }}$ as a parameter. Consequently, out of phase burst synchronization and bifurcation phenomena were observed. In the range of the amplitude $i_{\max }$ between 6.25 and 9.0 , bursts of RFNs are stably out of phase, such as shown in Fig. 3A, where $i_{\text {max }}=9.0$ and each burst contains three spike. In this case, the trajectory in the phase plane is very close to the threshold (Fig. 3A left), and thus this burst synchronization becomes unstable and a bifurcation occurs when $i_{\text {max }}$ is increased slightly further. When $i_{m a x}$ is around 10.7 , each burst contains two spikes through bursts of RFNs are still out of phase (Fig. 3B). In this range of $I_{m a x}, 1: 1$ out of phase synchronization coexists (Fig. 3C); when the initial phase difference between two RFN models is nearly $\pi$, the out of phase synchronization occurs. At $i_{\max }=14.0$, the trajectory in the phase plane is very close to the threshold, and thus another bifurcation occurs at this point. When $i_{\max }$ exceeds 14.0, 1:1 out of phase synchronization becomes stable. In addition, bursts of RFNs are roughly out of phase in the range of $i_{\max }$ from 9.1 to 10.6 through the bursts are quasi periodic.

Figure 4 shows trajectories in the $x_{1}-y_{1}$ and $y_{1}-y_{2}$ phase planes, and the symmetry we can find in Fig, $4 \mathrm{~B}$ indicates the out of phase synchronization of quasi periodic bursts. The stability of such out of phase synchronization could be analyzed by a similar method presented in [2]. 


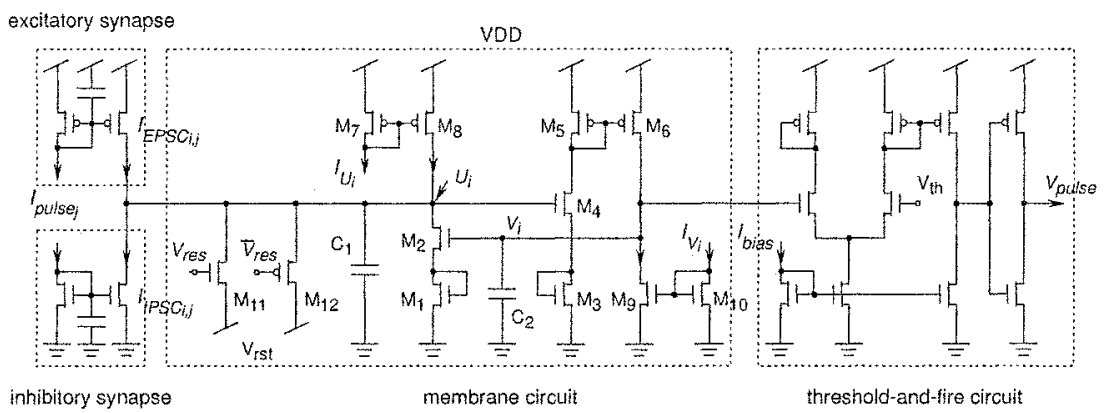

Fig. 5. Schematic of the resonate-and-fire neuron circuit. The circuit consists of a membrane circuit, a threshold-and-fire circuit, and excitatory and inhibitory synaptic circuits. The membrane circuit has the second-order dynamics containing two state variables $V_{i}$ (a current-like variable) and $V_{i}$ (a voltage-like variable), and it shows oscillatory behavior mediated and modulated by synaptic inputs.

\section{Circuit Implementation of the RFN model}

We designed the RFN model as an analog integrated circuit in our previous work [21]. Figure 5 shows the schematic of the RFN circuit, which consists of a membrane circuit, a threshold-and-fire circuit, and current-mirror integrators as excitatory and inhibitory synaptic circuits [4].

The membrane circuit was derived from the Volterra system for modeling prey-predator interactions to mimic the membrane dynamics of the RFN model by using the current-voltage relationship of subthreshold MOS FETs [21]. The dynamics of the membrane circuit are described as follows:

$$
\begin{aligned}
& C_{1} \frac{d U_{i}}{d t}=-g\left(U_{i}-V_{r s t}\right)+I_{i n}+\bar{I}_{U_{i}}-I_{0} \exp \left(\frac{\kappa^{2}}{\kappa+1} \frac{V_{i}}{V_{T}}\right) \\
& C_{2} \frac{d V_{i}}{d t}=I_{o} \exp \left(\frac{\kappa^{2}}{\kappa+1} \frac{U_{i}}{V_{T}}\right)-\bar{I}_{V_{i}}
\end{aligned}
$$

where the voltages $U_{i}$ and $V_{i}$ are state variables, $C_{1}$ and $C_{2}$ the capacitance, $g$ the total of the conductance of transistors, $\mathrm{M}_{11}$ and $\mathrm{M}_{12}, V_{r s t}$ the reset voltage, $\kappa$ the capacitive coupling ratio from the gate to the channel, $V_{T}$ the thermal voltage, and $I_{o}$ the pre-exponential current [4]. $I_{\text {in }}$ represents the summation of synaptic currents:

$$
I_{\mathrm{in}}=\sum_{j} I_{\mathrm{EPSC}_{i, j}}-\sum_{j} I_{\mathrm{PSSC}_{i, j}}
$$

where $I_{\mathrm{EPSC}_{i, j}}$ and $I_{\mathrm{IPSC}_{i, j}}$ represent the $i$-th post-synaptic currents through the $j$ th excitatory and inhibitory synaptic circuits. The currents, $\widetilde{I}_{U_{i}}$ through $\mathrm{M}_{8}$ and $\bar{I}_{V_{i}}$ through $\mathrm{M}_{9}$, are approximately described as follows: 


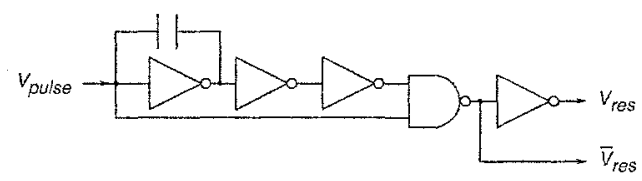

Fig. 6. Schematic of the delay-and-inverter circuit.

$$
\begin{aligned}
& \bar{I}_{U_{i}}=\alpha I_{U_{i}}\left(1+\frac{\mathrm{VDD}-U_{i}}{V_{E, p}}\right) \\
& \bar{I}_{V_{i}}=\beta I_{V_{i}}\left(1+\frac{V_{i}}{V_{E, n}}\right)
\end{aligned}
$$

where $I_{U_{i}}$ and $I_{V_{i}}$ are bias currents, VDD the power-supply voltage, $V_{E, p}$ and $V_{E, n}$ the Early voltage [4] for an nMOS FET and a pMOS FET, respectively, and $\alpha$ and $\beta$ the fitting constants.

The equilibrium point of the RFN circuit, $\left(U_{o}, V_{o}\right)$, can easily be obtained, and the stability of the point can also be analyzed by the eigenvalues of the Jacobian matrix of the circuit,

$$
J=\left[\begin{array}{ll}
-\frac{\alpha I_{U_{j}}}{V_{E}, p} & -\frac{\kappa^{2}}{\kappa+1} \frac{I_{V_{o}}}{V_{T}} \\
\frac{\kappa}{\kappa+1} \frac{Y_{U_{o}}}{V_{T}} & -\frac{\beta I_{V_{j}}}{V_{E, x_{i}}}
\end{array}\right]
$$

where $I_{U_{o}}$ and $I_{V_{o}}$ represent the equilibrium current at the equilibrium point. It is assumed that the leak conductance $g$ is zero. We used diode-connected transistors $\mathrm{M}_{1}$ and $\mathrm{M}_{3}$ to obtain small coefficients for $I_{U_{o}}$ and $I_{V_{o}}$, and short transistors having small Early voltages for $\mathrm{M}_{7}-\mathrm{M}_{10}$ to obtain large coefficients for $I_{U_{i}}$ and $I_{V_{i}}$. Consequently, the equilibrium point becomes a focus, and the membrane circuit exhibits damped oscillation in response to an input. In this case, the circuit dynamics is qualitatively equivalent to the membrane dynamics of the RFN model near the equilibrium point.

Inputs thorough synaptic circuits change the trajectory of the oscillation of the membrane circuit. If $V_{i}$ exceeds a threshold voltage $V_{\mathrm{th}}$, the thresholdand-fire circuit that consists of a comparator and an inverter generates a spike, i.e. a pulse voltage $V_{\text {pulse }}$, and the spike is fed into a delay-and-inverter circuit [9] with a mirror capacitance shown in Fig. 6. The delay-and-inverter circuit converts $V_{\text {pulse }}$ to reset voltages $V_{\text {res }}$ and $\bar{V}_{\text {res }}$ for reseting $U_{i}$ to a bias voltage $V_{\text {rst }}$. Pulse current, $I_{p u l s e, i}$, is generated as a result of switching by $V_{\text {res }}$, and given into the synaptic circuits. The behavior of the RFN circuit described above is qualitatively the same as that of the RFN model.

\section{Burst Synchronization in Pulse-Coupled RFN circuits}

We here demonstrate burst synchronization in two pulse-coupled RFN circuits using the circuit simulator, T-Spice Pro, with the model parameters for the 
A

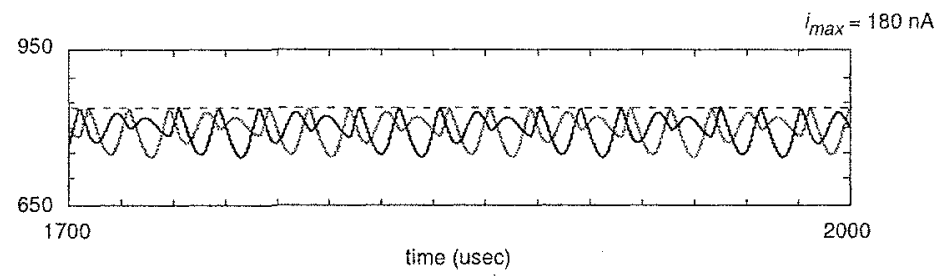

B

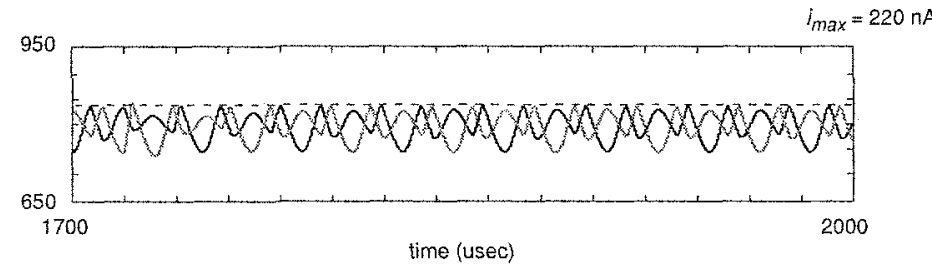

C

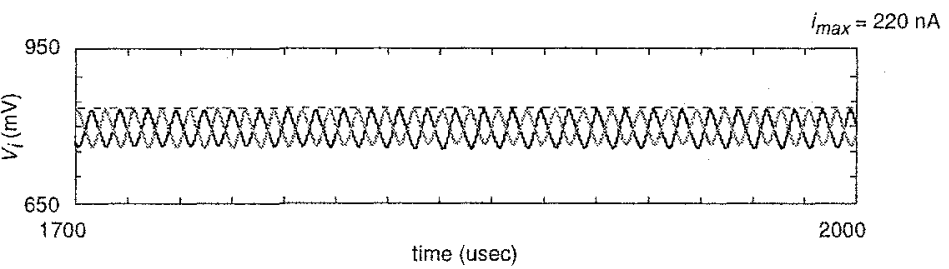

Fig. 7. Out of phase synchronization in two pulse-coupled resonate-and-fire circuits.

AMI 0.35- $\mu \mathrm{m}$ CMOS process. Through following simulations, we set the supply voltages at $\mathrm{VDD}=1.5 \mathrm{~V}, V_{\mathrm{th}}=835 \mathrm{mV}$, and $V_{\mathrm{rst}}=730 \mathrm{mV}$, the bias current at $I_{U_{i}}=14 \mathrm{nA}, I_{V_{i}}=10 \mathrm{nA}$, and $I_{\text {bias }}=250 \mathrm{nA}$, and the capacitance of the membrane circuit, the delay-and-inverter circuit, and the synaptic circuits, at $C_{1}=C_{2}=0.5 \mathrm{pF}, C_{3}=0.5 \mathrm{pF} . C_{\mathrm{p}}=0.02 \mathrm{pF}$, respectively.

Figure 7 shows out of phase synchronization of the two pulse-coupled RFN circuits. We determined circuit parameters so that the subthreshold behavior of the RFN circuit became qualitatively same as that of the RFN model with the parameters used in the previous section, and the period of the subthreshold oscillation was set at about $18.3 \mu \mathrm{s}$. We changed the maximal amplitude of the synaptic current $i_{\max }$ from $150 \mathrm{nA}$ to $250 \mathrm{nA}$. The synaptic currents were injected into each RFN circuit through five excitatory synaptic circuits for each firing. When $i_{\max }=180 \mathrm{nA}, 3: 3$ out of phase burst synchronization was observed (Fig. 7A). Such 3:3 burst synchronization became unstable around $i_{\max }=193 \mathrm{nA}$. Figure 7B shows $2: 2$ out of phase burst synchronization at $i_{\max }=220 \mathrm{nA}$. Such $2: 2$ out of phase burst synchronization coexists with $1: 1$ synchronization depending on the initial conditions (Fig. 7C). Figure 8 shows phase plane portraits of the RFN circuit during two types of the burst synchronization at a steady state. In spite of disturbance due to reseting pulse noise, both types of burst synchronization were maintained. These results show 
A

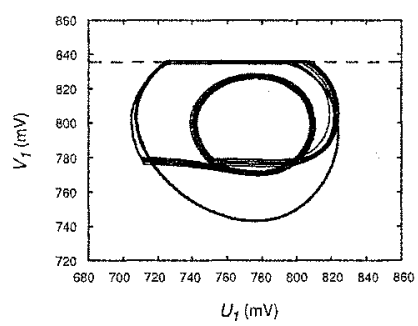

B

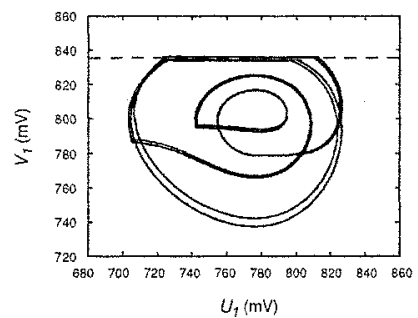

Fig. 8. Phase plane portraits of the RFN circuit at a steady state. (A) 3:3 out of phase burst synchrnozation. (B) 2:2 out of phase burst synchronization.

the stability of the out of phase burst synchronization in the two pulse-coupled RFN circuits. When out of phase burst synchronization became unstable, quasi periodic burst synchronization occurred. During autonomous firing, the firing frequency is larger than the natural frequency of the subthreshold oscillation of the RFN circuit. This induces the out of phase burst synchronization. Thus, the location of the reset value affects the stability of the out of phase burst synchronization since the reset value determines the firing frequency during autonomous periodic firing. In the above simulations, we fixed the parameters except for the maximal amplitude of the synaptic current. If we change the parameters, such as the threshold value and the bias currents, the stability of burst synchronization may change greatly. It should be noted that the bias currents change the equilibrium point and the natural frequency of the RFN circuit; consequently they also change the stability of the burst synchronization.

\section{Conclusions}

We have shown the out of phase burst synchronization in two pulse-coupled RFN model and its analog integrated circuit implementation. Through circuit simulations using SPICE, we have confirmed the stable region for out of phase burst synchronization. Such burst synchronization is of peculiar in the system of two pulse-coupled RFN circuits. As further considerations, we are going to study collective behavior in a large scale of coupled RFN circuits in view of both theoretical analysis and practical implementation.

\section{References}

1. E. M. Izhikevich, "Resonate-and-fire neurons," Neural Networks, vol. 14, pp. 883$894,2001$.

2. K. Miura and M. Okada, "Pulse-coupled resoante-and-fire neurons," Phys. Rev. E, vol. 70, 021914, 2004. 
3. C. A. Mead, Analog VLSI and neural systems, Addison-Wesley, Reading, 1989.

4. S.-C. Liu, J. Kramer, G. Indiveri, T. Delbruck, and R. Douglas, Analog VLSI: Circuits and Principles, MIT Press, 2002.

5. S.-C. Liu, J. Kramer, G. Indiveri, T. Delbruck, T. Burg, and R. J. Douglas, "Orientation-selective aVLSI spiking neurons," Neural Networks, vol. 14, no. 6-7, pp. 629-643, 2001.

6. M. Cheely and T. Horiuchi, "Analog VLSI models of range-tuned neurons in the bat echolocation system," EURASIP J. Appl. Signal Proc., vol. 7, pp. 649-658, 2003

7. S.-C. Liu and R. J. Douglas, "Temporal coding in a network of silicon integrateand-fire neurons," IEEE Trans. Neural Networks, Vol. 15, NO.5, pp. 1305-1314, 2004.

8. Y. Kanazawa, T. Asai, M. Ikebe, and Y. Amemiya, "A novel CMOS circuit for depressing synapse and its application to contrast-invariant pattern classification and synchrony detection," Int. J. Robotics and Automation, vol. 19, no. 4, pp. 206-212, 2004.

9. H. Tanaka, T. Morie, K. Aihara, "Associative memory operation in a Hopfieldtype spiking neural network with modulation of resting membrane potential," presented at Int. Symp. on Nonlinear Theory and its Applications, Bruges, Belgium, 2005.

10. G. Indiveri, E. Chicca and R. J. Douglas, "A VLSI array of low-power spiking neurons and bistable synapse with spike-timing dependent plasticity," IEEE Trans. Neural Networks, vol. 17, no. 1, pp. 211-221, 2006.

11. C. Rasche and R. H. R. Hahnloser, "Silicon synaptic depression," Biol. Cybern., vol. 84, pp. 57-62, 2001.

12. S.-C. Liu, "Analog VLSI circuits for short-term dynamic synapses," EURASIP J. Appl. Signal Proc, vol. 7, pp 620-628, 2003.

13. A. Bofill-i-Petit and A. F. Murray, "Synchrony detection and amplification by silicon neurons With STDP synapses, IEEE Trans. Neural Networks, Vol. 15, NO.5, pp. 1296-1304, 2004.

14. S. R. Schultz and M. A. Jabri, "Analogue VLSI integrate-and-fire neuron with frequency adaptation," Electronic Letters, vol. 31, no. 16, pp. 1357-1358, 1995.

15. K. A. Boahen, Retinomorphic Vision Systems: Reverse Engineering the Vertebrate Retina, Ph.D. thesis, California Institute of Technology, Pasadena CA, 1997.

16. A. van Schaik, "Building blocks for electronic spiking neural networks," Neural Networks, vol. 14, no. 6-7, pp. 617-628, 2001.

17. G. Indiveri, "A low-power adaptive integrate-and-fire neuron circuit," in proc. IEEE Int. Symp. Circ. Syst., 2003.

18. T. Asai, Y. Kanazawa, and Y. Amemiya, "A subthreshold MOS neuron circuit based on the Volterra system," IEEE Trans. Neural Networks, vol. 14, no. 5, pp. $1308-1312,2003$.

19. H. Nakano and T. Saito, "Grouping synchronization in a pulse-coupled network of chaotic spiking oscillators," IEEE Trans. Neural Networks, vol. 15, no. 5, pp. 1018-1026, 2004.

20. Y. Horio, T. Taniguchi and K. Aihara, "An Asynchronous Spiking Chaotic Neuron Integrated Circuit," Neurocomputing, Vol. 64, pp. 447-472, 2005.

21. K. Nakada, T. Asai, H. Hayashi, "A silicon resotate-and-fire neuron on the Volterra system," presented at Int. Symp. on Nonlinear Theory and its Applications, Bruges, Belgium, 2005. 